\section{An evaluation of senior house officer training in oral and maxillofacial surgery}

\author{
H. Stancliffe, ${ }^{1}$ R. Little, ${ }^{2}$ D. Keith ${ }^{3}$ and J. Durham ${ }^{4}$
}

IN BRIEF
- Understand current OMFS SHO
perceptions of training in UK.
Understand and be able to identify why
SHOs in your unit may be dissatisfied
with their training.
Identify areas for improvement within
your own training programmes for
OMFS SHOs.

The senior house officer ( $\mathrm{SHO}$ ) is a training post but there is concern over whether service commitments are impinging on this. The European Working Time Directive (EWTD) and Foundation Programme have recently been introduced to try and improve working lives and training quality respectively. Aim To examine the current perceptions of SHO training in relation to the recommended standards in the UK, and to compare the level of training with data sets from the survey in 2002 (Keith and Durham 2002). Method A questionnaire using Likert, dichotomous and free text responses was posted to all SHOs in oral and maxillofacial surgery (OMFS) in the UK. The questionnaire was almost identical to that used in a previous national survey (Keith and Durham 2002). The data set from the previous survey was available thereby allowing statistical comparisons to be made. Results An estimated response rate of $56 \%$ was achieved $(n=228)$. Forty-eight percent of respondents felt undergraduate BDS training was inadequate for their job, and the longer individuals had been qualified the less they felt out of their depth $(r=0.452, p<0.0001)$. These findings were consistent with the previous survey. Formal training in medical examination of patients had been provided to 58\%, which was slightly less than previously (64\%). Over half of those responding had regular supervised local anaesthetic minor oral surgery sessions (55\%), and outpatient clinics with a designated trainer (54\%). A minority of trainees had not had any appraisal (27\%). The majority of respondents (79\%) stated their rota was EWTD-compliant. Conclusion Improvements have been made but the introduction of a national structured programme could help standardise training.

\section{INTRODUCTION}

Over the last two decades the working practices and education of junior doctors have changed..$^{1-3}$ These changes were made in an attempt to improve junior doctors' working lives and decrease the potential for errors due to lack of rest. ${ }^{4-10}$ The 'New Deal'1 and more recently the European Working Time Directive (EWTD) $)^{2,3}$ both placed restrictions on the number of hours an individual can work and imposed minimum rest requirements. An individual can choose to opt out of the EWTD, but the rest break requirements are not negotiable. The

\footnotetext{
'Oral and Maxillofacial Department, Newcastle Dental Hospital, Richardson Road, Newcastle upon Tyne,

NE2 4AZ; ${ }^{2}$ Community Dental Department, Arthur's Hill Clinic, Douglas Terrace, Newcastle upon Tyne, NE4 6BT; ${ }^{3}$ Oral and Maxillofacial Department, Sunderland Royal Hospital, Kayll Road, Sunderland, SR4 7TP; ${ }^{4 *}$ Oral and Maxillofacial Department, School of Dental Sciences, Framlington Place, Newcastle University, Newcastle upon Tyne, NE2 4AB

${ }^{*}$ Correspondence to: Dr Justin Durham Email: j.a.durham@ncl.ac.uk
}

\section{Refereed Paper}

Accepted 10 June 2011

DOI: 10.1038/sj.bdj.2011.572

${ }^{\circledR}$ British Dental Journal 2011; 211: 75-80
Directive was endorsed into British law on 1 October 1998, but was incorporated into working practices in three stages: a limitation to 58 hours per week from August 2004, 56 hours from August 2007 and finally 48 hours from August 2009.

There has been documented concern within the medical profession as a whole that the changing in working patterns necessary to be EWTD compliant might negatively affect both patient care and training opportunities for junior doctors. ${ }^{11,12}$ Concurrent to the significant change in working patterns there have been two large changes in postgraduate training for doctors and dentists. ${ }^{13,14}$ Given the recent changes and consequential concerns over decreased training opportunities the aim of this evaluation was to examine the current perceptions of oral and maxillofacial senior house officer (OMFS SHO) training in relation to the recommended standards in the UK, ${ }^{15}$ and to compare the level of training with a previously published data set from the period before the EWTD. ${ }^{16}$

\section{AIM}

To examine the current perceptions of SHO training in relation to the recommended standards in the UK, and to compare the level of training with data sets from the survey in 2002. ${ }^{16}$

\section{METHOD}

To allow statistical and qualitative comparison with the previously published data set ${ }^{16}$ the 29 item ad hoc questionnaire used in the current evaluation largely mirrored that used in 2002 with only minor modifications used to make it valid for the current day. Modifications included enquiries about EWTD compliance, Foundation Programme, career pathways and new qualifications. The questionnaire consisted of a mixture of free-text, dichotomous and Likert responses. The topic areas covered included: qualifications, opinions about training, weekly timetable, working hours, and on call commitments.

The participants' job-related confidence was assessed by asking how often they felt out of depth, overwhelmed or lacking in 


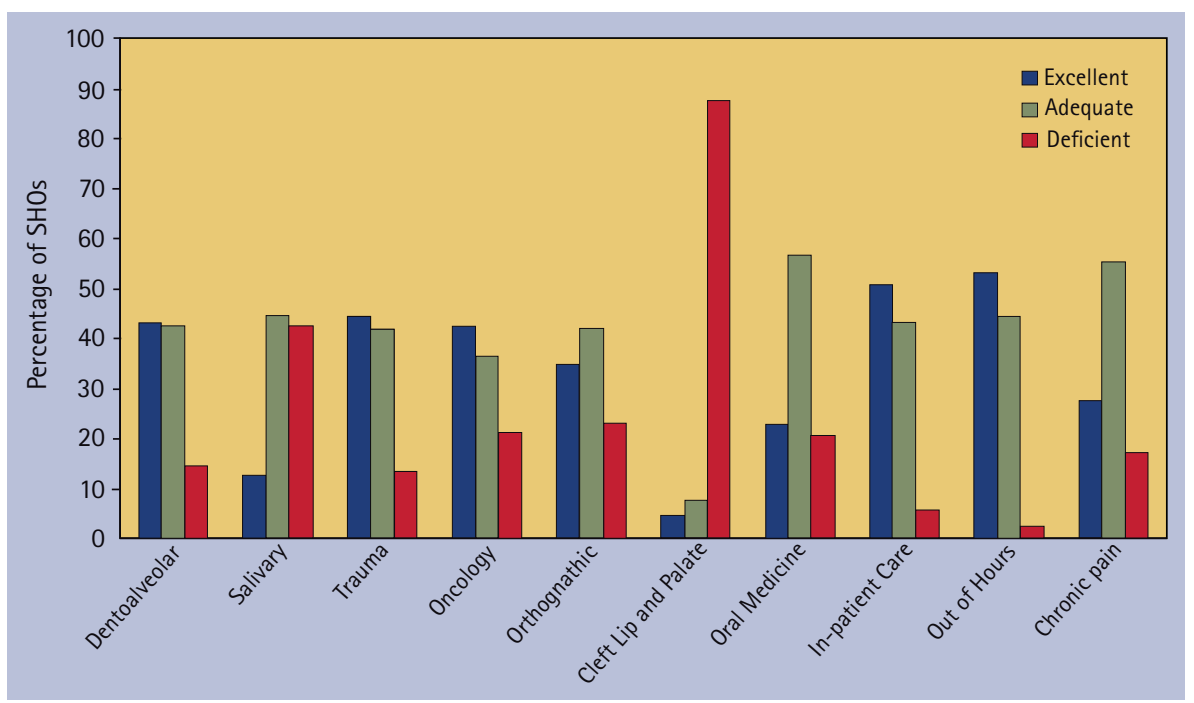

Fig. 1 Opinions of Senior House Officers about exposure to aspects of oral and maxillofacial surgery in 2010

confidence with patient management $(0$, always feeling out of depth; 5 never feeling out of depth). Participants' opinions on the level of exposure to specific surgical procedures (Fig. 1) were examined using a three-point scale of excellent, adequate, and deficient.

The anonymous questionnaires were posted, with a covering letter, to all UK Oral and Maxillofacial Units. These units were identified from the British Association of Oral and Maxillofacial Surgeons' register of units. Each unit was contacted by telephone in October 2009 to ascertain the number of SHOs they had in permanent posts. We estimated that there were 409 occupied training posts. Questionnaires were posted to all units during November 2009 and only those in a permanent post were invited to participate. All units were reminded to return the questionnaires via telephone in January 2010. The data from the returned questionnaires from 2010 were entered into a database, crosschecked, and then analysed with SPSS (Version 17, New York, USA) using simple descriptive statistics, chi-square, Mann-Whitney test and Spearman Rho.

\section{RESULTS}

A total of 228 questionnaires were returned with an estimated response rate of 56\%. The group consisted of $46 \%$ male and $54 \%$ female.

Table 1 shows the number of years the respondents had been qualified (mean 3.5 S.D. 2.67). The mean number of years spent in OMFS as an SHO was 1.0 (S.D. 0.97). Only two respondents (1\%) had a medical undergraduate qualification and the majority (70\%) had completed part of or a full postgraduate qualification, with 44\% having completed MFDS or equivalent (Table 2). Currently 34\% of OMFS SHOs are part of the Foundation Programme.

Figure 1 shows the sample's opinions on the level of exposure their post provides in the varying aspects of oral and maxillofacial surgery. The majority of respondents $(88 \%)$ felt that their exposure to cleft lip and palate was deficient and 43\% of SHOs found their exposure to salivary gland surgery deficient. Oral medicine, chronic pain and orthognathic surgery have predominantly adequate exposure only.

Those responding had a week consisting of a mean of: 3.9 (S.D 1.61) operating sessions (LA and GA); 2.9 (S.D 1.25) outpatient clinic sessions; 0.9 (S.D 0.75) pre-admission clinic sessions. Operating sessions could be either local or general anaesthesia. In the case of the former, 55\% of respondents had regular local anaesthetic minor oral surgery sessions with a designated trainer. A minority operated as the most 'senior operator' for general anaesthetic cases (25\%). Outpatient clinics were also conducted with a designated trainer, undertaking teaching, for over half of the respondents (54\%). Less than half $(45 \%)$ of respondents reported a weekly session for administration or audit tasks. The majority (86\%) of these individuals, however, reported having to sacrifice this session because of work commitments.

Opinions were divided on the adequacy of undergraduate BDS training for the post, with $48 \%$ of the opinion that it was

\begin{tabular}{|c|c|}
\hline Years qualified & Number of SHOs (\%) \\
\hline$>1-<2$ & $80(35)$ \\
\hline$>2-<3$ & $51(22)$ \\
\hline$>3-<4$ & $27(12)$ \\
\hline$>4-<5$ & $16(7)$ \\
\hline$>5-<6$ & $18(8)$ \\
\hline$>6-<7$ & $9(4)$ \\
\hline$>7-<8$ & $4(2)$ \\
\hline$>8-<9$ & $8(4)$ \\
\hline$>9-<10$ & $6(2)$ \\
\hline$>10$ & $8(4)$ \\
\hline
\end{tabular}

Table 2 Postgraduate qualifications of Senior House Officers in 2010

\begin{tabular}{l|l}
\hline Qualifications & Number of SHOs (\%) \\
\hline FDS part 1 & $1(1)$ \\
\hline FDS/FFD & $1(1)$ \\
\hline MFDS part A & $15(7)$ \\
\hline MFDS part B & $7(3)$ \\
\hline MFDS & $19(9)$ \\
\hline MFDS part 1 & $15(7)$ \\
\hline New MFDS & $54(27)$ \\
\hline MJDF part 1 & $34(17)$ \\
\hline MJDF & $26(13)$ \\
\hline MSC & $9(4)$ \\
\hline Other & $23(11)$
\end{tabular}

not adequate. There was a weak correlation between increased time as a SHO and positive perception of the adequacy of BDS training for the SHO post $(\mathrm{r}=0.130$, $\mathrm{p}<0.05)$.

Confidence at work was examined in the questionnaire and the analysis of the data suggested: the longer individuals had been qualified the less frequently they felt out of depth ( $r=0.452, p<0.0001)$, and the greater amount of time spent as a SHO the more confident they became $(\mathrm{r}=0.481$, $\mathrm{p}<0.001)$. There was also a weak correlation between feeling out of depth and not having consultant clinics with a designated trainer $(\mathrm{r}=0.135, \mathrm{p}<0.05)$.

Over half of respondents (57\%) had received formal training in medical examination of patients. Ninety percent had been 
shown venous cannulation, 63\% urethral catheterisation, 64\% nasogastric (NG) tube placement, and 42\% arterial blood gas sampling. Since 2002 the only skill to show a significant decrease in training is the placement of a urinary catheter $\left(\chi^{2}=5.067, p<0.05\right)$ (Table 3). Ninety-eight percent have attended at least one of the following: hospital induction course (79\% of total sample), trauma course (24\% of total sample), postgraduate cardiopulmonary resuscitation (CPR) course (68\% of total sample), or a 'dentist on the ward' course ( $72 \%$ of total sample). Attendance at formal training sessions for SHOs has significantly increased since 2002 for 'dentist on the ward' courses $\left(\chi^{2}=43.158\right.$, $\mathrm{p}<0.001)$, hospital induction courses $\left(\chi^{2}\right.$ $=4.685, \mathrm{p}<0.05)$, and postgraduate $\mathrm{CPR}$ courses $\left(\chi^{2}=17.218, p<0.001\right)$. However, it has significantly decreased for trauma courses $\left(\chi^{2}=5.592, p<0.05\right)$. At the time of the questionnaire $27 \%$ of the sample had yet to receive any appraisal. Fiftyfive percent of the sample reported that they had not had the recommended appraisal at two weeks. Over half (52\%) of the individuals had however been appraised in the post by two months and the majority (62\%) had been appraised by six months.

Figure 2 demonstrates that 63\% of SHOs were between a 1 in 5 and 1 in 8 on call rota, and 65\% were resident on call. Residency while on call has significantly decreased from 87\% in 2002 ( $\chi^{2}=33.926$, $\mathrm{p}<0.001)$. Eighty-two percent of respondents, who are resident while on call, stated that their rota complies with the EWTD. However, in reality up to $56 \%$ may be non-compliant, provided that they do not share their out of hours care with another specialty (Fig. 3). Just under half of those responding (48\%), who work on call, work a consecutive week of nights and 34\% of SHOs, who have a divided night shift pattern, work three and four nights separately.

Figure 4 shows the on call banding of the sample. Only 57\% felt that the band allocated was a fair reflection of the work intensity of their post, which is significantly less than in $2002\left(\chi^{2}=12.577, p<0.05\right)$. However, of those allocated Bands $1 \mathrm{~A}$ and $1 \mathrm{~B}$, associated with less remuneration, $44 \%$ did not think the banding was a fair reflection of the work intensity of the post.

The future career plans of the sample

\begin{tabular}{|c|c|c|}
\hline Précis of question & $\begin{array}{l}\text { Number of SHOs in } \\
\text { responding positively } \\
\text { in } 2002(\%)\end{array}$ & $\begin{array}{l}\text { Number of SHOs in } \\
\text { responding positively } \\
\text { in } 2010(\%)\end{array}$ \\
\hline Training in arterial blood gases & $111(49)$ & $96(42)$ \\
\hline Training in nasogastric tube & $134(59)$ & $146(64)$ \\
\hline Training in venous cannulation & $192(85)$ & $205(90)$ \\
\hline Training in catheterisation & $165(73)$ & $144(63)^{*}$ \\
\hline Attended dentist on ward course & $94(41)$ & $164(72)^{* *}$ \\
\hline Attended ATLS & $76(33)$ & $53(23)^{*}$ \\
\hline Attended CPR & $110(48)$ & $153(68)^{* *}$ \\
\hline Attended hospital induction & $160(70)$ & $180(79)^{*}$ \\
\hline Had formalised Medical Examination training in post & $144(64)$ & $131(57)$ \\
\hline Have a supervised MOS List & $105(47)$ & $126(55)$ \\
\hline Have supervised outpatient clinics & $137(61)$ & $123(54)$ \\
\hline Have a SHO led GA List & $67(30)$ & $58(25)$ \\
\hline Are you Resident on call & $194(87)$ & $144(65)^{* *}$ \\
\hline On call banding fair & $159(73)$ & $128(57)^{* *}$ \\
\hline
\end{tabular}

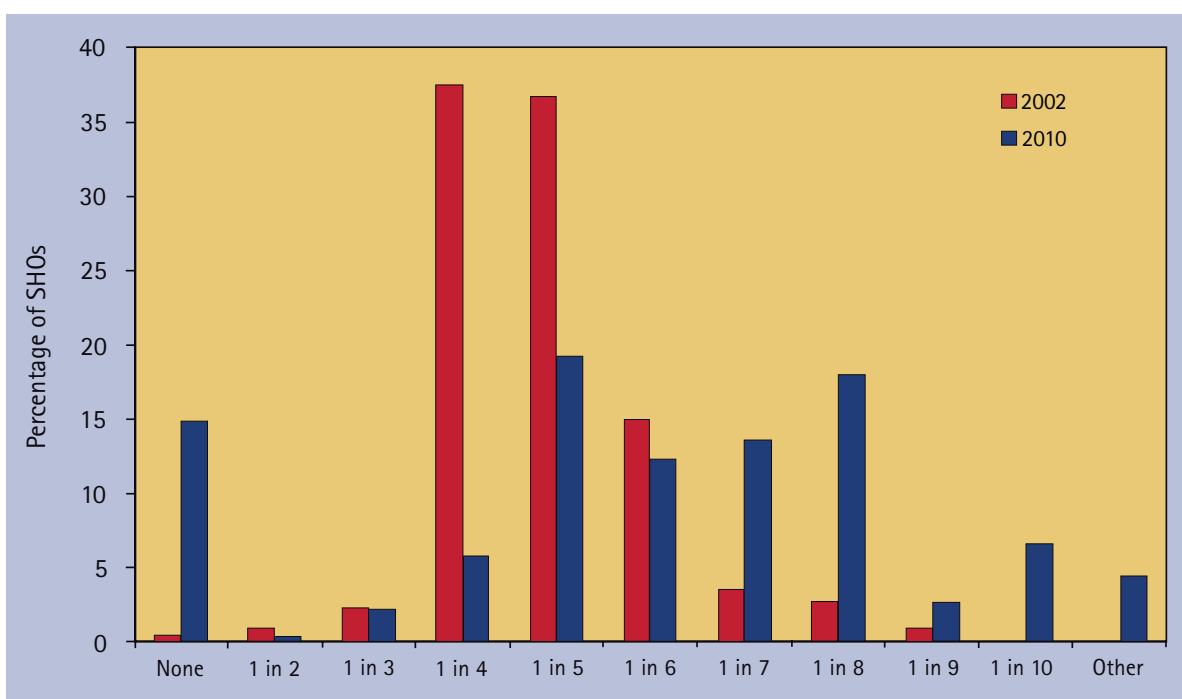

Fig. 2 Distribution of Senior House officer on call working patterns between 2002 and 2010

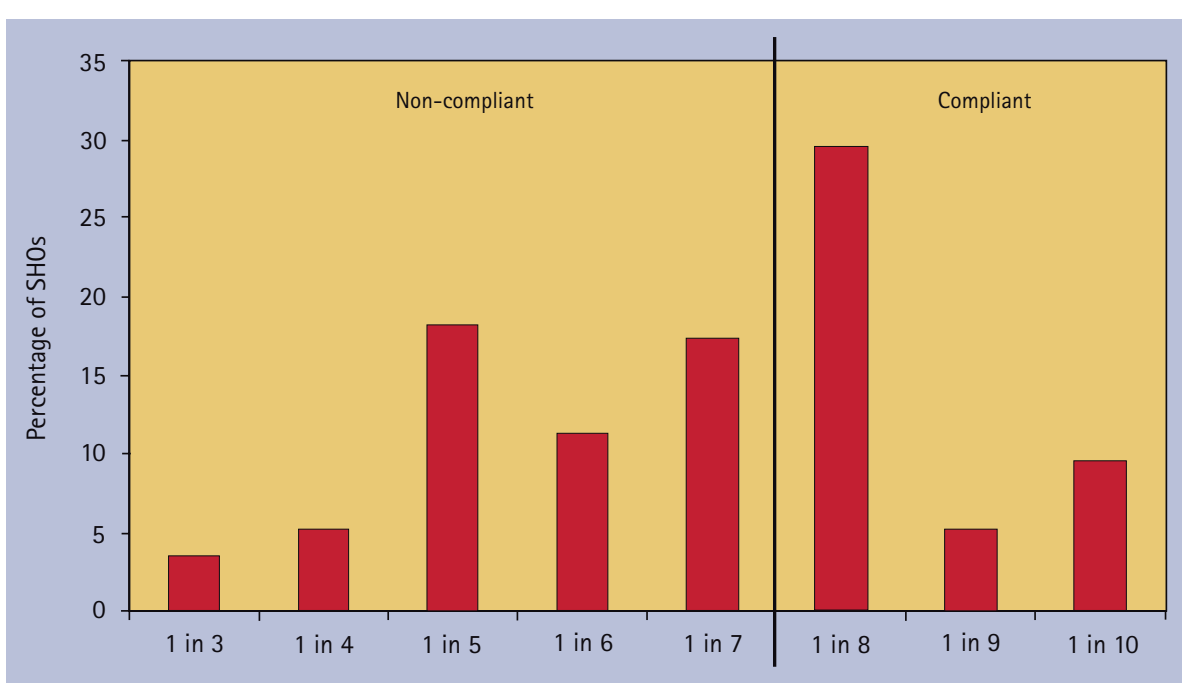

Fig. 3 EWTD compliance of SHOs resident on call in 2010 
are shown in Figure 5 with the majority being undecided.

\section{DISCUSSION}

The apparent lack of a centralised database of all of the Maxillofacial SHOs currently in post across the United Kingdom made it impossible to accurately calculate the total number of SHOs available for the questionnaire. Even when the individual OMFS units were contacted directly there appeared sometimes to be uncertainty about which SHOs were currently in post and this was also true when some of the educational deaneries were contacted. In 2002 there were an estimated 305 occupied training posts, which has now increased by 104 . This increase would appear to be due to increased numbers of SHOs in some units as there has been no increase in the total number of OMFS units. A similar number of questionnaires were, however, returned in 2002 and 2010 thereby allowing a relatively straightforward statistical comparison.

The questionnaire used in this study was limited to the perceptions of the SHOs in the posts about their workload and perceptions can fluctuate dependent on a number of factors. A more accurate method would be obtaining rotas from each unit or logbooks from the SHOs to show the variation in their workload across the different centres. The design of the questionnaire could also be improved by tailoring it towards guidelines for SHOs stated by COPDEND, to allow for a direct comparison. However, this would have affected the ability to make direct comparisons with the 2002 cohort.

Overall SHOs are still generally satisfied with the variety of exposure to the different aspects of OMFS that they receive; the majority of responses lying between excellent and adequate. The most striking result was that $88 \%$ felt that their exposure to cleft lip and palate was deficient but this is unavoidable due to the work being limited to specialist centres. Exposure to salivary gland surgery may be deficient due to this work being carried out less frequently than other procedures or may reflect that other specialties, such as ENT and plastic surgery, are carrying out this work. The number of orthognathic cases may be limited due to the specialist nursing care required post-operatively, and oral medicine

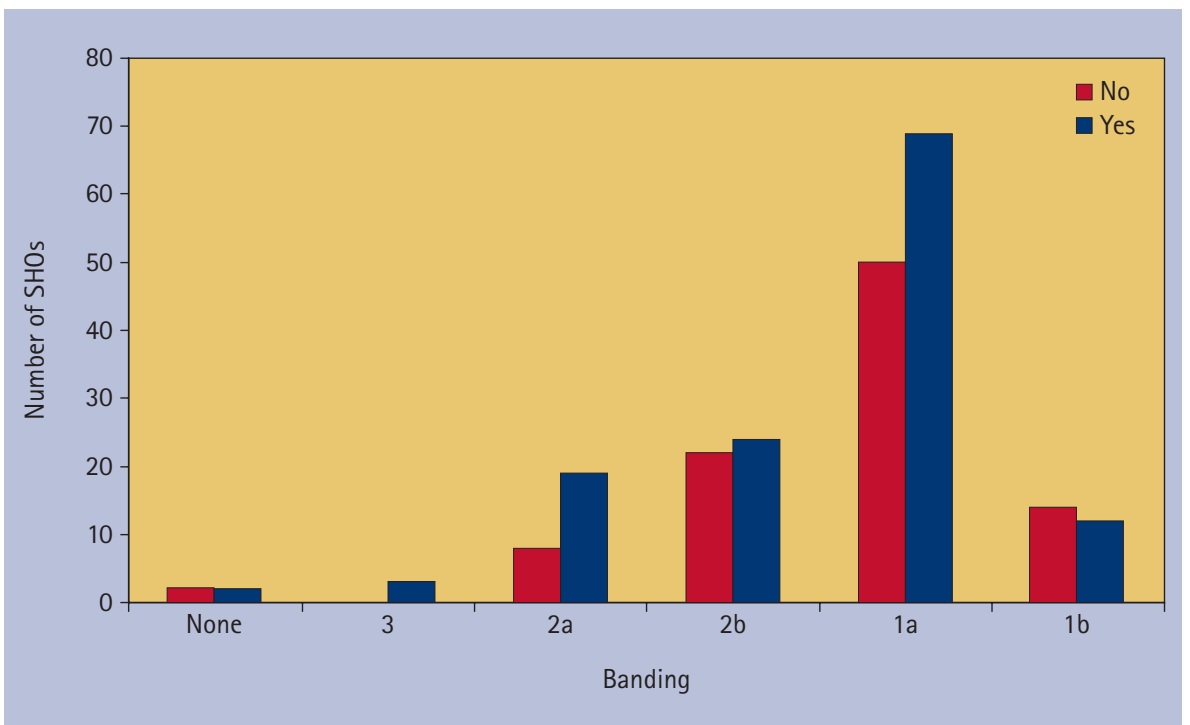

Fig. 4 Satisfaction with on call banding and banding distribution in 2010

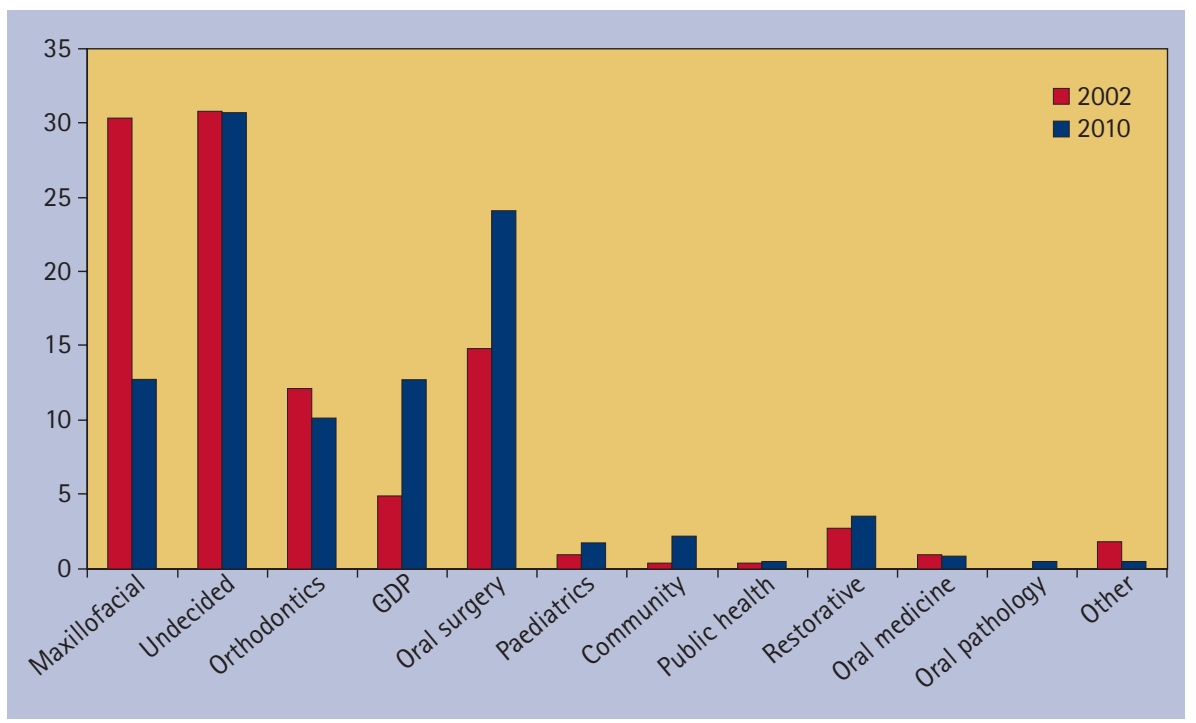

Fig. 5 Career plans of Senior House Officers in 2002 and 2010

and chronic pain may be referred to specialist clinics.

Although the satisfaction over the exposure to dentoalveolar surgery is relatively high, the percentage of trainees meeting the requirements of supervised minor oral surgery list with a designated trainer is only 55\%. This lack of supervision may affect the quality of the training and the progression of skills development. Trainees also may not realise the benefits of a supervised list, having never experienced one, and may not develop a realistic interpretation of their skill level either through lack of feedback or the constant exposure to simple cases.

Training experience remains similar between 2002 and 2010, however, the guidelines from COPDEND ${ }^{15}$ are still not being met. Overall the daytime situation may not have appreciably worsened since the introduction of EWTD. Given the decrease in the SHO on call commitments from 2002 to 2010, it appears that these have been reduced in preference to daytime duties in order to meet the EWTD. The skills being lost may therefore be associated with the out of hours care of patients which are likely to provide a large amount of experiential and observational learning, and decision-making practice. The increase in number of SHO posts has been necessary to comply with EWTD, but this may negatively affect both patient care and training opportunities for junior doctors.

The learning curve as a new OMFS SHO can be particularly steep. The skills required of a SHO differ greatly from those required in dental practice. Although 48\% felt that their undergraduate training was 
inadequate, this is subject to recall bias, especially as $43 \%$ had graduated more than three years ago and so their perceptions may have altered. A small minority (4\%) had also qualified more than ten years previously; this may be due to stagnation within their career progression or the decision to undertake a SHO post at a later stage in their career.

As the majority of respondents did not hold a medical qualification the skills expected to be acquired of $\mathrm{SHOs}^{15}$ of venepuncture, urinary catheterisation, arterial blood gas sampling and the placement of nasogastric (NG) tubes should have a high emphasis within their training as they are less likely to have been gained as an undergraduate. There has also been a slight decrease in the number of SHOs who have received formal training in the medical examination of patients. As it is often the SHO's responsibility to clerk a patient for theatre it is disconcerting that less than half suggest that they have not been taught how to ensure a patient is medically fit for theatre, as it is conceivable that they may be the only person to perform a review of systems before a general anaesthetic being administered.

Although COPDEND specifies a structured timetable for SHOs to follow, it does not indicate the skills they should gain throughout their training. Throughout the country, the experience of SHOs could vary dramatically. The introduction of a structured training programme with a list of learning objectives and clinical skills to be achieved is necessary to demonstrate a satisfactory level of competence and to standardise training nationwide. ${ }^{17}$ This could be assessed by ensuring a set number of observed competencies are undertaken under supervision by middle grades or consultants, until competence is achieved in each clinical area. Possible areas for assessment might include: venous cannulation, surgical extraction of wisdom teeth, and medical examination of patients. Without this standardisation and assessment of training, it is conceivable that SHOs at present could stay at this career level for an unsatisfactory length of time, stagnating and not improving their skills. In contrast, in a more structured programme, by achieving all the necessary competencies, a SHO would be demonstrably ready to progress to their next stage of training. With the introduction of the Foundation Programme consideration may need to be given to a modified curriculum for Foundation trainees who hold SHO posts, as their training time is limited to six months and their experience may differ from their colleagues.

Standards are being met with the number of operating sessions being carried out on average on a weekly basis by SHOs. ${ }^{15}$ Achieving one bleep-free training session per week in any acute surgical trauma unit is likely to provide a constant challenge. Such a session, however, is a good opportunity to teach or reinforce SHOs' clinical skills and knowledge. Suggestions from the Royal College of Surgeons of England to try to accommodate these sessions include: duplicating structured training twice a week to ensure all trainees can attend, rotating the teaching to maximise attendance, or arranging whole days of teaching on a less frequent basis. ${ }^{18}$ All of these suggestions have their limitations and would be difficult to achieve in most cases as another grade of staff would have to act as first on call.

Over the past 19 years there has been a significant decrease in the number of hours worked by doctors. ${ }^{1,2}$ This reduction is reflected in the change in on call commitments with $63 \%$ working between a 1 in 5 and a 1 in 8 rota. The most frequent amount of remuneration is now 50\% of basic pay, previously $80 \% .{ }^{15}$ Consequently there has been a significant decrease in how fair SHOs feel their banding is in relation to the work intensity of their post from 73\% (2002) to 57\% (2010). It is uncertain what the SHOs' understanding of EWTD is as 79\% believe that their rotas are compliant when many are on seemingly incompliant rotas. The consensus may be that the rotas comply over each cycle but do not comply week on week; this is because $48 \%$ work a consecutive week of nights, which is strongly discouraged by EWTD. ${ }^{19}$

With regards to appraisal, educational supervisors are not meeting their targets, although figures have improved since 2002. Without guidance from their supervisors SHOs will not have developed their learning objectives or targets. It is during this time that SHOs gain guidance about their careers and how to achieve their aims. The lack of appraisal could account for the 31\% with no known plan.

As with the 2002 cohort, a large proportion of SHOs remain undecided about their career plans. However, the mean time spent as an SHO has decreased from 2002 to 2010. This could indicate that the introduction of Modernising medical careers ${ }^{13}$ has started to succeed in decreasing the time it takes to complete a training pathway and overcome the bottleneck in the training identified by Harris and Ferreira. ${ }^{20}$ Since the introduction of the more structured oral surgery pathway ${ }^{21}$ in 2008 , the number of SHOs wanting to pursue an OMFS career has decreased from 30\% in 2002 to $13 \%$ in 2010. Conversely, the percentage of SHOs wishing to follow a career in oral surgery has increased. This is most likely due to the introduction of the training pathway in oral surgery and the ability to pursue it while being singularly qualified. OMFS consultants or newly appointed/ existing consultants in oral surgery will have to provide the future training for these posts as they continue to develop. This shift in career aspirations alongside the increase in university fees may lead to a decrease in candidates applying for OMFS, which may become more apparent in the coming years. How this will affect the profession overall remains to be seen.

\section{CONCLUSION}

The 2010 data suggest that the COPDEND guidelines are not being met. However, when the two data sets are compared, it can be seen that improvements have been made in a time of upheaval with introduction of EWTD. It is difficult to quantify training experience as some skills are learnt through experiential and observational learning, which is especially true for out of hours care.

The balance between the training needs of the SHO and the service requirements of a department will always be a challenge to meet. However, the introduction of a nationwide structured training programme would standardise SHO training to ensure all trainees become safe and competent clinicians in OMFS, and discourage stagnation at the SHO level.

1. Department of Health. Hours of work of doctors in training: the new deal. London: Department of Health, 1991.

2. The European Working Time Directive. UK notification of derogation for doctors in training. Department of Health, 2009 


\section{EDUCATION}

3. Council Directive 93/104/EC. Official Journal of the European Community 1993; L307: 18-24.

4. Williamson A M, Feyer A M. Moderate sleep deprivation produces impairments in cognitive and motor performance equivalent to legally prescribed levels of alcohol intoxication. Occup Environ Med 2000; 57: 649-655.

5. Feyer A M. Fatigue: time to recognize and deal with an old problem. Br Med J 2001; 322: 908-909.

6. Dawson D, Reid K. Fatigue, alcohol and performance impairment. Nature 1997; 388: 235.

7. Taffinder N J, McManus I C, Gul Y, Russell R C G Darzi A. Effect of sleep deprivation on surgeons dexterity on laparoscopy simulator. Lancet 1998; 352: 1191.

8. BMA Junior Doctors Committee. Implications for health and safety of junior doctors' working arrangements. London: British Medical Association, 2000.

9. Royal College of Physicians. Designing safer rotas for junior doctors in the 48 hour week. London: RCP,

\section{6.}

10. Knauth P. Speed and direction of shift rotation J Sleep Res 1995: 4(Suppl 2): 41-46.

11. BMA survey of junior doctor working arrangements and the EWTD, 2008

12. Royal College of Physicians of Edinburgh and Scottish Academy. UK survey on the early impact of the EWTD, 2009

13. Department of Health. Modernising medical careers: the next steps. The future shape of Foundation, Specialist and General Practice Training Programmes. 15 April 2004.

14. Conference of Postgraduate Dental Deans and Directors. A Curriculum for UK Dental Foundation Programme Training, 2006.

15. Conference of Postgraduate Dental Deans and Directors, Faculty of Dental Surgery of the Royal College of Surgeons of England. A guide for skills required for house officers and senior house officers in the dental specialities in England and Wales.
London, 2002.

16. Keith D J W, Durham J. Senior house officer training in oral and maxillofacial surgery: a national survey. Br Dent J 2003; 195 (Suppl): 23-28.

17. NHS Scotland. Dentally qualified senior house officers in oral and maxillofacial surgery units: Report on requirements for education, training and clinical supervision. Scotland, 2003.

18. Working Time Directive - Implications and Practical Suggestions to Achieve Compliance. Joint Royal College of Anaesthetists and Royal College of Surgeons of England WTD 2009 Project.

19. The Royal College of Surgeons of England. Working Time Directive 2009: Meeting the challenge in surgery. June 2008 .

20. Harris E, Ferreira P. Training senior house officers. BrMed J (Suppl) 1997; 314: 692

21. The Royal College of Surgeons of England. Oral surgery training - a modern flexible programme. July 2008 